\title{
Research on Adaptive Friction Compensation of Digital Hydraulic Cylinder Based on LuGre Friction Model
}

\author{
Shouling Jiang, ${ }^{1}$ Kun Zhang $\mathbb{D}^{2}{ }^{2}$ Hui Wang, ${ }^{1}$ Donghu Zhong, ${ }^{1}$ Jinpeng Su $\mathbb{D}^{2,3}$ \\ and Zengkai Liu $\mathbb{D}^{2}$ \\ ${ }^{1}$ School of Mechanical Engineering, Liaoning Technical University, No. 88, Yulong Road, Fuxin, Liaoning 123000, China \\ ${ }^{2}$ Shandong Provincial Key Laboratory of Robotics and Intelligent Technology, Shandong University of Science and Technology, \\ No. 579, Qianwangang Road, Qingdao, Shandong 266590, China \\ ${ }^{3}$ State Key Laboratory of Mechanical System and Vibration, Shanghai Jiao Tong University, Dongchuan Road 800, \\ Shanghai 200240, China \\ Correspondence should be addressed to Kun Zhang; zhangkunliaoning@163.com
}

Received 12 July 2020; Revised 5 February 2021; Accepted 5 May 2021; Published 24 May 2021

Academic Editor: Franck Poisson

Copyright (C) 2021 Shouling Jiang et al. This is an open access article distributed under the Creative Commons Attribution License, which permits unrestricted use, distribution, and reproduction in any medium, provided the original work is properly cited.

\begin{abstract}
This paper aims to eliminate nonlinear friction from the performance of the digital hydraulic cylinder to enable it to have good adaptive ability. First, a mathematical model of a digital hydraulic cylinder based on the LuGre friction model was established, and then a dual-observer structure was designed to estimate the unobservable state variables in the friction model. The Lyapunov method is used to prove the global asymptotic stability of the closed-loop system using the adaptive friction compensation method. Finally, Simulink is used to simulate the system performance. The simulation results indicate that the addition of adaptive friction compensation control can effectively reduce system static error, suppress system limit loop oscillation, "position decapitation," "speed dead zone," and low-speed creep phenomena, and improve the overall performance of the digital hydraulic cylinder. The control method has practical application value for improving the performance index of the digital hydraulic cylinder.
\end{abstract}

\section{Introduction}

Digital hydraulic cylinders have great application potential because of their advantages of simple structure, low cost, high control accuracy, and suitable for application in harsh working environments [1]. In recent years, digital hydraulic cylinders have been applied to digital excavating robots, loading robots, and so on. The digital hydraulic cylinder is essentially a nonlinear system [2] in which nonlinear friction will cause the system position static difference, reduce system stability, increase speed zero-crossing distortion, and limit cycle oscillation and other phenomena. Therefore, the friction of the system must be compensated and suppressed to improve the performance of digital hydraulic cylinders [3].

Friction compensation can be divided into nonmodel and model-based compensation. Nonmodel friction compensation control includes $\mathrm{PD}$, robust, and sliding mode control. Ren et al. [4] used a high-gain PD controller to suppress friction nonlinearity. The differential term in the PD controller increases system damping, which can improve the low-speed tracking performance of the system to a certain extent and suppress the crawling phenomenon; however, it will still produce a steady-state error. Simply increasing the system gain can only reduce the steady-state error, but it does not eliminate it, causing the stability of the system to deteriorate. Wang and Chai [5] designed an adaptive fuzzy compensation PD controller based on $\mathrm{PD}$, which can effectively improve the tracking performance of the system. Hu et al. [6] used the disturbance observer-based robust control for friction compensation. This method can improve the stability of the system. However, because it is a linear compensation, the degree of compensation for friction nonlinearity depends on the bandwidth of the filter. The increase in bandwidth is limited by factors, such as mechanical 
resonance in the actual system, which is the exact problem with this compensation method. Feng and Li et al. [7-9] designed a sliding mode variable structure control friction compensator. Although the linear sliding mode determines the deviation between the system state and the given trajectory converges exponentially, that is, the system state continues to approach the given trajectory, the given trajectory cannot be reached forever and the control quantity has a jitter phenomenon. Model-based adaptive compensation method for online friction compensation is generally used as a solution for most researchers. The model-based compensation method usually estimates the parameters of the friction model online and adds the friction compensation term to the control algorithm to perform online compensation for the friction [10]. Liu and Wang et al. [11, 12] proposed a decomposition control to achieve friction compensation, but the friction model uses a static friction model, and the effect after system compensation is not very ideal. The more the friction model can reflect the dynamic and static characteristics of the friction force in the system, the better the friction compensation effect. Friction models, such as Coulomb + viscous friction, exponential friction, and Karnopp are static friction models, which make it impossible to predict the dynamic friction behavior characteristics. Meanwhile, the Dhal model, LuGre model, and Bliman-Sorine are all dynamic friction models. The LuGre model is the latest achievement in this field and can describe the dynamic and static characteristics of friction well [13-15]. Xiang et al. [16] proposed an adaptive friction compensation method based on the LuGre model. The backstepping method was used to design an adaptive friction compensation controller. This method could effectively suppress the adverse effects of friction interference on the servo system. Wang et al. [17] proposed an adaptive friction compensation method based on the observer/filter structure based on the LuGre model, which was verified by the simulation to improve the tracking performance of the system. Han et al. [18] adopted the sliding mode variable structure control and neural network control to estimate the friction model parameters and found that it could better suppress system disturbance and friction nonlinearity and improve the positioning accuracy and robustness of the system. Mobayen and Tchier [19] proposed a new state-feedback stabilization control technique for a class of uncertain chaotic systems with Lipschitz nonlinearity conditions.

Chang et al. [20] considered the random uncertainty in-vehicle system model, and based on methods $H_{\infty}$ and $L_{2}-H_{\infty}$, they proposed a variety of flexible controllers that considered random uncertainty to make the closedloop system stable in the sense of mean square and provided $H_{\infty}$ and $L_{2}-H_{\infty}$ performance indicators. Wang et al. [21] investigated the problem of adaptive neural output-feedback tracking control for a class of switched uncertain nonlinear systems in the nonstrict-feedback structure with average dwell time. To address the issue originating from the nonstrict-feedback structure, an effective adaptive law is introduced by adopting the properties of NNs. The effect of the dead zone on control performance is restricted by designing a special adaptive law in the last step of the backstepping design frame. The stability of the closed-loop system is proved by the average dwell time approach and Lyapunov stability theory; $\mathrm{Li}$ et al. [22] studied a class of multi-input-output nonlinear systems with unknown dead zone and limited output. They used neural network observers to approximate all unmeasured states and used DSC and backstepping control and Lyapunov methods to design observer-based adaptive neural control strategies, which ensure that the output of the switching system can track the target trajectory in a small range. Zhang et al. [23] proposed a disturbance observer based on regression disturbance fuzzy neural network for the tracking control problem of uncertain nonlinear systems. The modified method can be applied to the synchronization control of chaotic gyro systems. Wei and Zhang [24] focused on a class of nonlinear strict-feedback systems with interference by designing a nonlinear interference observer and proposed an anti-interference control method based on nonlinear interference observer and backstepping to compensate for interference, which ensured that all signals of the closedloop system are semiglobal and eventually uniformly bounded.

In this paper, based on the ideas of reference [25], the adaptive friction compensation algorithm is established for the characteristics of digital hydraulic cylinders. The dualobserver structure is designed using the most complete dynamic friction model, i.e., LuGre model, and the parameters in the friction model are estimated online. In online compensation for nonlinear friction, the controller is divided into a friction compensation item and a robust control item. The friction model compensation item is used to suppress nonlinear friction. The robust control item can improve the robust performance of the entire system when an external disturbance occurs. The Lyapunov method is used to prove the global asymptotic stability of the closedloop system using the adaptive friction compensation method. Finally, Simulink is used to simulate the system performance.

\section{Digital Hydraulic Cylinder Dynamic Analysis}

The main links of the digital hydraulic link and the system schematic diagram are shown in Figure 1.

\subsection{Feedback Nut and Ball Screw Conversion Mechanism} Model. The valve opening is a compound movement in space under the combined action of the feedback nut and the ball screw. That is, the input angle of the stepping motor is transformed into the displacement $k_{1} \theta_{v}$ of the spool through the feedback nut. After the valve port is opened, the hydraulic cylinder causes the ball screw to rotate at the same time, and the negative feedback displacement of the stroke spool is $-k_{1} \theta_{v}$. The absolute displacement of the final synthetic spool is $x_{v}$. The following equation can be used to describe its movement process [26]: 


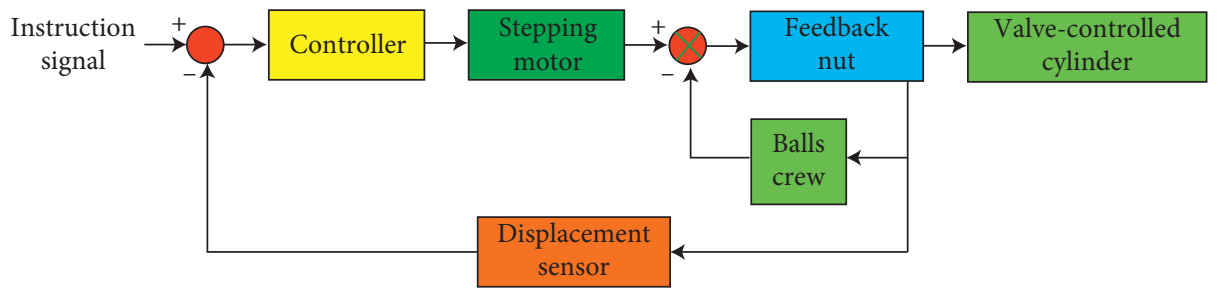

Figure 1: Schematic diagram of a digital hydraulic cylinder system.

$$
\left\{\begin{array}{l}
x_{v}=k_{1}\left(\theta_{v}-\theta_{s}\right), \\
\theta_{v}=u \cdot k_{4}, \\
\theta_{s}=k_{2} x_{p}, \\
k_{1}=\frac{p_{1}}{2 \pi}, \\
k_{2}=\frac{2 \pi}{p_{2}},
\end{array}\right.
$$

where $x_{v}$ is the actual displacement of the spool, $x_{p}$ is the displacement of the hydraulic cylinder piston, $\theta_{v}$ is the inherent step angle of the stepper motor, $\theta_{s}$ is the feedback rotation angle of the ball screw, $p_{1}$ is the feedback nut lead, $p_{2}$ is the ball screw lead, $k_{1}$ is the feedback nut gain, $k_{2}$ is the ball screw gain, and $u$ is the controller output.

2.2. Stepper Motor Model. The input signal of the stepper motor is the number of pulses, and the output is the angular displacement, which can be approximated using a proportional component. Its gain is expressed by $k_{4}$.

2.3. Feedback Loop Model. The displacement sensor converts the displacement signal output by the digital hydraulic cylinder into an electrical signal and feeds it back to the input terminal. Its frequency response is much greater than the frequency response of the system. Therefore, it can be considered as a proportional link and expressed by gain $k_{3}$.

2.4. Hydraulic Cylinder Friction Model. The LuGre friction model can better describe the static and dynamic friction effects of the system. Using the LuGre model to investigate the friction force $F_{f c}$, the following can be used:

$$
\left\{\begin{array}{l}
\frac{\mathrm{d} z}{\mathrm{~d} t}=\dot{x}_{p}-\frac{\left|\dot{x}_{p}\right|}{g\left(\dot{x}_{p}\right)} z, \\
F_{f c}=\sigma_{0} z+\sigma_{1} \dot{z}+\sigma_{2} \dot{x}_{p}, \\
\sigma_{0} g\left(\dot{x}_{p}\right)=F_{c}+\left(F_{s c}-F_{c}\right) e^{-\left(\dot{x}_{p} v_{s k}\right)^{2}},
\end{array}\right.
$$

where $z$ is the average deformation of bristle, $F_{C}$ represents the Coulomb friction, $F_{S C}$ refers to the maximum static friction, $\sigma_{0}$ is the bristle stiffness coefficient, $\sigma_{1}$ refers to the bristle viscous damping coefficient, $\sigma_{2}$ is the system viscous damping coefficient, and $v_{S K}$ is the Stribeck critical velocity.

2.5. Valve Control Cylinder Link. The two throttle windows of the spool are assumed to be matched and symmetrical, the charge oil pressure is constant, and the spill pressure is constantly zero. The available valve port linearized flow equation is as follows:

$$
q_{L}=k_{q} x_{v}-k_{c} p_{c}
$$

Assuming that the connecting pipeline between the valve and the hydraulic cylinder is short and thick, the pressure loss and pipeline dynamics in the pipeline can be ignored. The oil temperature and the volumetric elastic modulus are constant. The internal and external leakages of the hydraulic cylinder are both laminar flow. The available hydraulic cylinder flow continuity equation is as follows:

$$
q_{L}=A_{h} \frac{\mathrm{d}_{x_{p}}}{\mathrm{~d}_{t}}+\frac{v_{0}}{\beta_{e}}+\frac{\mathrm{d}_{p_{c}}}{\mathrm{~d}_{t}}+c_{t p} p_{c} .
$$

The balanced equation of the output force of the hydraulic cylinder and the external load force is as follows:

$$
A_{h} p_{c}=m_{p} \frac{\mathrm{d}_{x_{p}}^{2}}{\mathrm{~d}_{t^{2}}}+F_{f c}+F_{L}+\Delta(x, z, t),
$$

where $q_{L}$ is the load flow, $k_{q}$ is the flow gain, $k_{C}$ is the flowpressure coefficient, $p_{C}$ is the control chamber pressure, $c_{t p}$ is the total leakage coefficient of the hydraulic cylinder, $p_{s}$ is the charge oil pressure, $A_{h}$ is the effective working area of the hydraulic cylinder, $v_{0}$ refers to the effective volume of the hydraulic cylinder, $\beta_{e}$ represents the elastic modulus of the oil volume, $m_{p}$ is the mass of the piston and its components, and $F_{L}$ is the external load force.

The goal of designing the controller $u$ is to make the tracking error of the closed-loop control system approach zero infinitely. Before designing the controller, the following assumptions are made about the system: uncertainty $\Delta(x, z, t) \leq \delta(t) f(t), \delta(t)$ is a known function and $f(t)$ is an unknown bounded function, assuming $|f(t)| \ll f_{d}$.

\section{Adaptive Friction Compensation}

Because the three parameters, $\sigma_{0}, \sigma_{1}$, and $\sigma_{3}$, in the LuGre model will change due to external factors such as 
temperature and material wear, it has been established that an adaptive control algorithm needs to be identified online to compensate for nonlinear friction.

The following equation is obtained from equations (3) and (4):

$$
p_{c}=\frac{K_{1} K_{4} K_{q}}{K_{c e}} u-\frac{K_{1} K_{2} K_{q}}{K_{c e}} x_{p}-\frac{A_{h}}{K_{c e}} \dot{x}_{p}-\frac{V_{0}}{\beta_{e} K_{c e}} \dot{p}_{c} .
$$

Bring equations (2) and (6) to equation (5) to obtain the following equation:

$$
m_{p} \ddot{x}_{p}=a u-b x_{p}-c \dot{x}_{p}-d \dot{p}_{c}-\sigma \dot{x}_{p}-\sigma_{0} z+\sigma_{1} \frac{\left|\dot{x}_{p}\right|}{g\left(\dot{x}_{p}\right)} z+\Delta(x, z, t)-F_{L}
$$

where $\quad a=\left(K_{1} K_{4} K_{q} A_{h} / K_{c e}\right), \quad b=\left(K_{1} K_{2} K_{q} A_{h} / K_{c e}\right)$, $c=\left(A_{h}^{2} / K_{c e}\right), \sigma=\sigma_{1}+\sigma_{2}$, and $K_{c e}=k_{c}+c_{t p}$.

Establish a systematic error equation as follows:

$$
\begin{aligned}
& e_{1}=k_{3} x_{p}-r, \\
& e_{2}=\dot{e}_{1}+k e_{1}, \\
& e_{2}=k_{3} \dot{x}_{p}-x_{2 e q},
\end{aligned}
$$

where $x_{2 e q}=r-k e_{1}, k$ is a constant greater than zero, and $e_{1}$ is the position tracking error.

$$
\dot{e}_{2}=k_{3} \ddot{x}_{p}-\dot{x}_{2 e q} \text {. }
$$

Bring equation (9) to equation (7) to obtain equation

$$
\frac{m_{p}}{K_{3}} \dot{e}_{2}=\left(a u-b x_{p}-c \dot{x}_{p}-d \dot{p}_{c}-\sigma \dot{x}_{p}-\sigma_{0} z+\sigma_{1} \frac{\left|\dot{x}_{p}\right|}{g\left(\dot{x}_{p}\right)} z\right)-\frac{m_{p}}{K_{3}} \dot{x}_{2 e q}+\Delta(x, z, t)-F_{L}
$$

Because the average deformation $z$ of the bristle in the LuGre model is not measurable, a state observer is required to estimate the state variable $z$ online. The two-state observers are as follows:

$$
\left\{\begin{array}{c}
\frac{\mathrm{d} \widehat{z}_{0}}{\mathrm{~d} t}=\dot{x}_{p}-\frac{\left|\dot{x}_{p}\right|}{g\left(\dot{x}_{p}\right)} \widehat{z}_{0}+\tau_{0}, \\
\frac{\mathrm{d} \widehat{z}_{1}}{\mathrm{~d} t}=\dot{x}_{p}-\frac{\left|\dot{x}_{p}\right|}{g\left(\dot{x}_{p}\right)} \widehat{z}_{1}+\tau_{1}, \\
z-\widehat{z}_{0}=\widetilde{z}_{0}, \\
z-\widehat{z}_{1}=\widetilde{z}_{1},
\end{array}\right.
$$

where $\quad(\mathrm{d} z / \mathrm{d} t)-\left(\mathrm{d} \widehat{z}_{0} / \mathrm{d} t\right)=(\mathrm{d} z / \mathrm{d} t)-\left(\mathrm{d} \bar{z}_{0} / \mathrm{d} t\right)$ $(\mathrm{d} z / \mathrm{d} t)-\left(\mathrm{d} \widehat{z}_{1} / \mathrm{d} t\right)=(\mathrm{d} z / \mathrm{d} t)-\left(\mathrm{d} \bar{z}_{1} / \mathrm{d} t\right)$.

Substituting equations (12) and (13) into equation (11), we obtain the following equation:

$$
\left\{\begin{array}{l}
\frac{\mathrm{d} \widetilde{z}_{0}}{\mathrm{~d} t}=\frac{\left|\dot{x}_{p}\right|}{g\left(\dot{x}_{p}\right)} \widetilde{z}_{0}-\tau_{0}, \\
\frac{\mathrm{d} \widetilde{z}_{1}}{\mathrm{~d} t}=-\frac{\left|\dot{x}_{p}\right|}{g\left(\dot{x}_{p}\right)} \widetilde{z}_{1}-\tau_{1} .
\end{array}\right.
$$

Because $\sigma, \sigma_{1}$, and $\sigma_{2}$ are all unknown parameters, they are replaced with estimated values. In the formula, $\tau_{0}$ and $\tau_{1}$ are the dynamic terms of the observer.

$$
\begin{aligned}
& \tau_{0}=e_{2}, \\
& \tau_{1}=-\frac{\left|\dot{x}_{p}\right|}{g\left(\dot{x}_{p}\right)} e_{2} .
\end{aligned}
$$

Let the adaptive robust controller of the system be

$$
u=u_{1}+u_{2}
$$

where $u_{1}$ represents the friction model compensation term and $u_{2}$ is the robust control term.

The control law of the friction compensation term is

$$
u_{1}=\frac{1}{a}\left(-k_{s} e_{2}+b x_{p}+c \dot{x}_{p}+d \dot{p}_{c}+\widehat{\sigma} \dot{x}_{p}+\widehat{\sigma}_{0} \widehat{z}_{0}-\widehat{\sigma}_{1} \frac{\left|\dot{x}_{p}\right|}{g\left(\dot{x}_{p}\right)} \widehat{z}_{1}\right)+F_{L} \text {, }
$$

where $\widehat{\sigma}$ and $\widehat{\sigma}_{1}$ are the estimated values of $\sigma$ and $\sigma_{1}$.

$$
\begin{gathered}
\widehat{\sigma}=\sigma-\widetilde{\sigma}, \\
\widehat{\sigma}_{1}=\sigma_{1}-\widetilde{\sigma}_{1} .
\end{gathered}
$$

Bring equations (18) into (19) to obtain the following equation: 


$$
\frac{m_{p}}{K_{3}} \dot{e}_{2}=-k_{s} e_{2}-\widetilde{\sigma} \dot{x}_{p}-\left(\sigma_{0} \widetilde{z}_{0}+\widetilde{\sigma}_{0} \widetilde{z}_{0}\right)+\left(\sigma_{1} \widetilde{z}_{1}+\widetilde{\sigma}_{1} \widetilde{z}_{1}\right) \frac{\left|\dot{x}_{p}\right|}{g\left(\dot{x}_{p}\right)}+a u_{2}+\Delta(x, z, t)+F_{L} .
$$

To overcome the system uncertainty caused by uncertain system parameters and external interference, the design satisfies the following equations:

$$
\begin{gathered}
e_{2}\left(-\widetilde{\sigma} \dot{x}_{p}-\left(\sigma_{0} \widetilde{z}_{0}+\widetilde{\sigma}_{0} \widehat{z}_{0}\right)+\left(\sigma_{1} \widetilde{z}_{1}+\widetilde{\sigma}_{1} \widehat{z}_{1}\right) \frac{\left|\dot{x}_{p}\right|}{g\left(\dot{x}_{p}\right)}+a u_{2}+\Delta(x, z, t) \leq \varepsilon_{0}+\varepsilon_{1} f_{d}\right) \\
e_{2} a u_{2} \leq 0
\end{gathered}
$$

Therefore, the design robust control term $u_{2}$ is as follows:

$$
u_{2}=-\frac{1}{4 a}\left(\frac{3}{\varepsilon_{0}} h_{0}^{2}+\frac{3}{\varepsilon_{0}} h_{1}^{2}+\frac{3}{\varepsilon_{0}} h_{2}^{2}+\frac{3}{\varepsilon_{0}} f(x)^{2}\right) e_{2},
$$

where $h_{0} \geq\|\Psi\|_{2}\left\|\Phi_{\max }-\Phi_{\min }\right\|_{2}, \quad h_{1} \geq \sigma_{0 \max }\left(z_{\max }-z_{\min }\right)$, $h_{2} \geq \sigma_{1 \max }\left(z_{\max }-z_{\min }\right) \quad\left(\left|\dot{x}_{p}\right| / g\left(\dot{x}_{p}\right)\right), \Psi=\left[\sigma_{0}, \sigma_{1}, \sigma\right]$, and $\Phi^{T}=\left[-\bar{z}_{0},\left(\left|\dot{x}_{p}\right| / g\left(\dot{x}_{p}\right)\right) \bar{z}_{1}, \dot{x}_{p}\right]$.

Let the Lyapunov function be $V(x, t)$ as follows:

$$
V(x, t)=\frac{1}{2} \frac{m_{p}}{K_{3}} e_{2}^{2}+\frac{1}{2} \sigma_{0} \widetilde{z}^{2}{ }_{0}+\frac{1}{2} \sigma_{1} \widetilde{z}^{2}{ }_{1}+\frac{1}{2} \widetilde{\sigma}^{2}+\frac{1}{2} \widetilde{\sigma}_{0}^{2}+\frac{1}{2} \widetilde{\sigma}_{1}^{2} \geq 0 .
$$

The adaptive laws for setting $\sigma, \sigma_{1}$, and $\sigma_{2}$ are as follows:

$$
\begin{aligned}
& \dot{\hat{\sigma}}_{1}=-e_{2} \dot{x}_{p}, \\
& \dot{\hat{\sigma}}_{0}=-e_{2} \widehat{z}_{0}, \\
& \dot{\hat{\sigma}}_{1}=e_{2} \widehat{z}_{1} \frac{\left|\dot{x}_{p}\right|}{g\left(\dot{x}_{p}\right)} .
\end{aligned}
$$

Differentiate $V(x, t)$ and bring equations (25)-(27) to obtain the following equation:

$$
\dot{V}(x, t)=-k_{s} e_{2}^{2}-\left(\sigma_{0} \widetilde{z}_{0}^{2}+\sigma_{1} \widetilde{z}_{1}^{2}\right) \frac{\left|\dot{x}_{p}\right|}{g\left(\dot{x}_{p}\right)} .
$$

Because $g\left(\dot{x}_{p}\right) \geq 0$ and $k_{s}>0, \dot{V}(x, t) \leq 0$. According to Lyapunov's second theorem, the closed-loop digital hydraulic cylinder with adaptive robust friction compensation is globally progressively stable.

\section{Simulation and Analysis}

A friction compensation simulation test is performed on the digital hydraulic cylinder based on the LuGre friction model based on the adaptive friction compensation controller designed above.

Figure 2 is a simulation block diagram of adaptive friction compensation, and Figure 3 is a system simulation model built using MATLAB/Simulink simulation software. The main parameters of the digital hydraulic cylinder studied in this paper are as follows: the effective area of the hydraulic cylinder is $A_{h}=0.005 \mathrm{~m}^{2}$, initial volume is $V_{0}=5 \times 10^{-4} \mathrm{~m}^{3}$, gradient of the valve port area is $w=84.8 \mathrm{~mm}$, the total mass of the piston moving parts is $m_{p}=500 \mathrm{~kg}$, the system oil supply pressure is $p_{s}=7 \mathrm{MPa}$, the feedback nut lead is $p_{1}=1.5 \mathrm{~mm}$, ball screw lead is $p_{2}=20 \mathrm{~mm}$, feedback coefficient is $k_{3}=0.5$, stepper motor driver gain is $k_{4}=18000$, the maximum static friction is $F_{S C}=800 \mathrm{~N}$, Coulomb friction is $F_{S}=400 \mathrm{~N}$, and Stribeck speed $V_{S K}=0.1(\mathrm{~m} / \mathrm{s})$.

4.1. Steady-State Limit Cycle Oscillation. Considering the step signal input, given the input position signal $r=5 \times 10^{-5}$, the gain of the adaptive control law is $k_{s}=10$ and $k=200$. A step of external interference is added during the system running $0.5 \mathrm{~s}$. The jump signal has a value of $100 \mathrm{~N}$.

The piston displacement curve with PID control and adaptive control law is shown in Figure 4. The enlarged curve in Figure 4(a) shows that the system with PID control has a stability limit cycle. If the system part suffers from wears or the temperature is affected by the operation, the original system will become an unstable system. The system is in a critically stable state. Figure 4(b) shows that the static difference of the system after adding adaptive friction compensation becomes zero, and therefore, adding adaptive friction compensation can effectively eliminate the limit cycle oscillation phenomenon of the system and improve the tracking accuracy of the system. Figures 4(a) and 4(b) also show that the response speed of the system with adaptive friction compensation has been improved considerably. The adaptive friction compensation strategy designed in this paper can effectively reduce the system static error caused by friction nonlinearity and the stability limit cycle phenomenon, but the response speed of the system decreased.

4.2. Low-Speed Crawl. After considering the slope input, a slope with a speed of $5 \times 10^{-5} \mathrm{~m} / \mathrm{s}$ is used as the system input, and the position tracking errors added with PID control and 


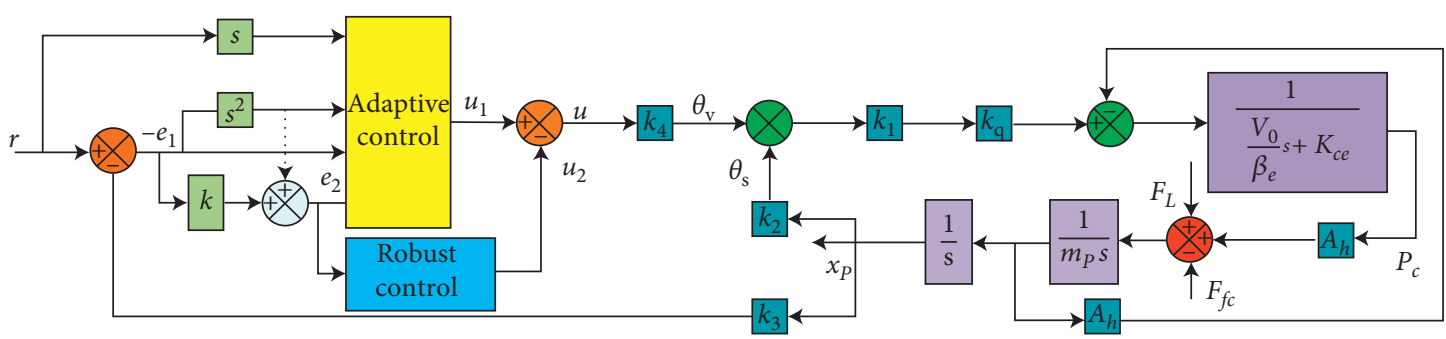

FIGURE 2: Simulation block diagram of adaptive friction compensation based on LuGre.

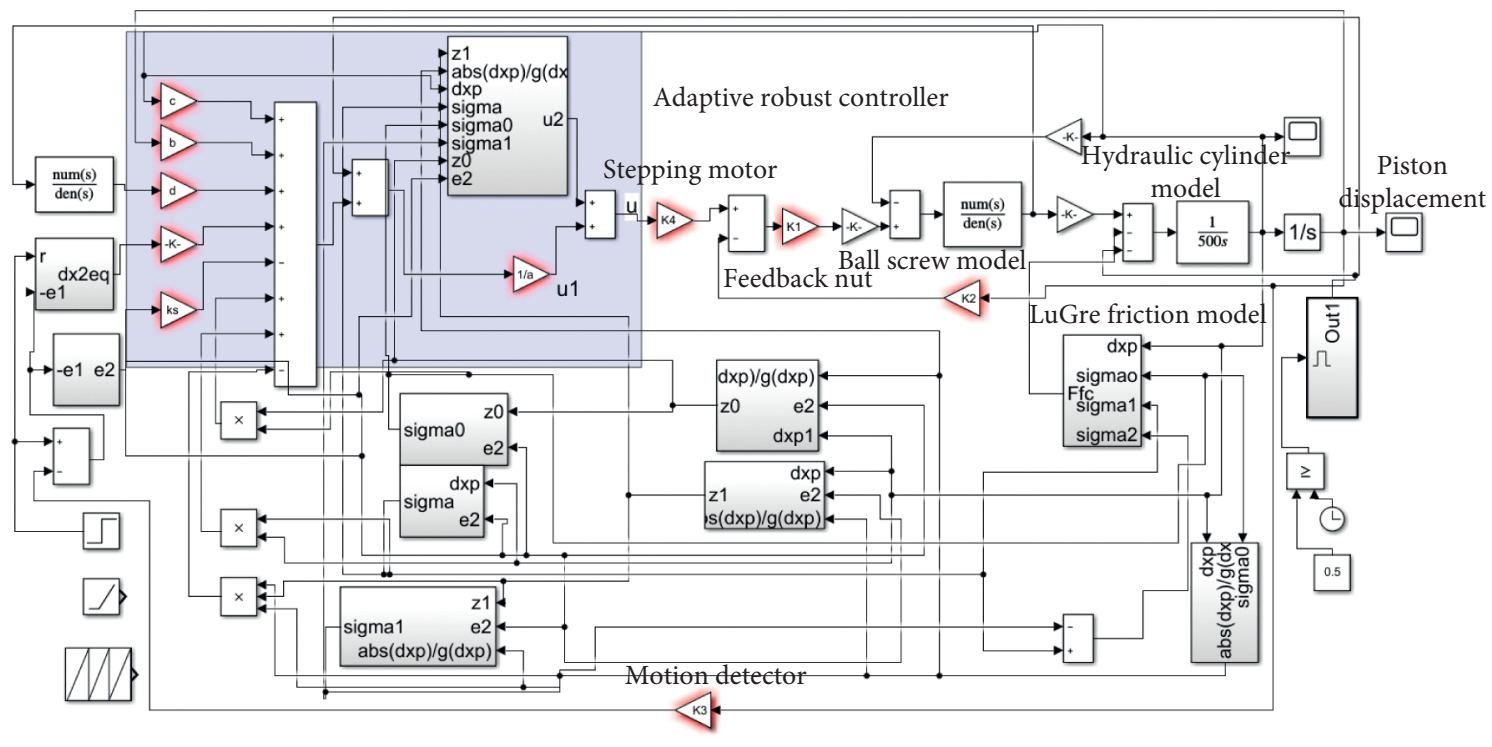

FIGURE 3: Simulation program of adaptive friction compensation control.

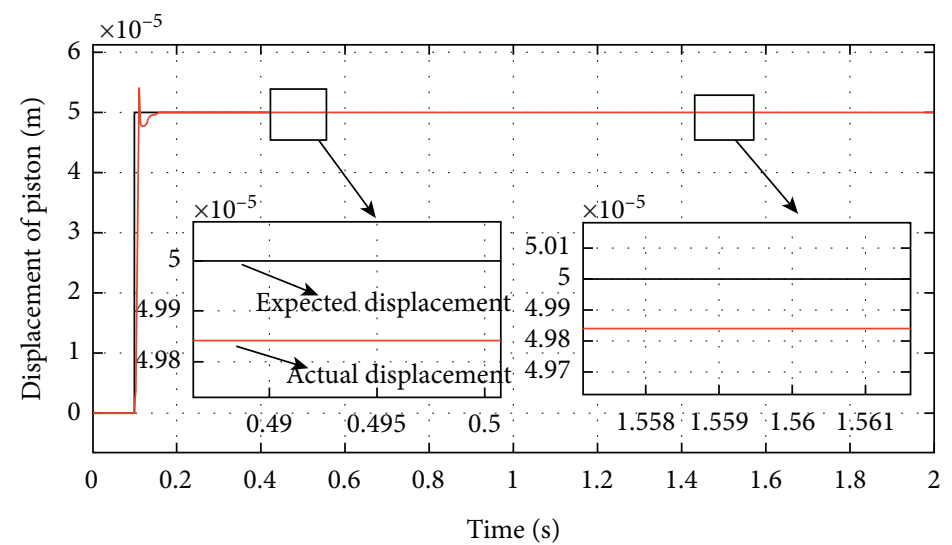

(a)

Figure 4: Continued. 


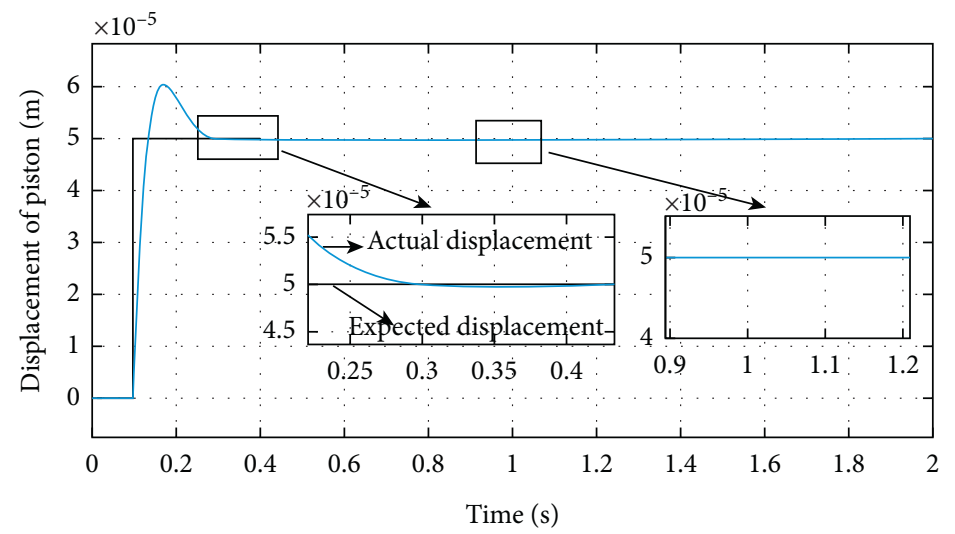

(b)

FIGURE 4: Piston displacement: (a) no adaptive friction compensation system added; (b) add adaptive friction compensation system.

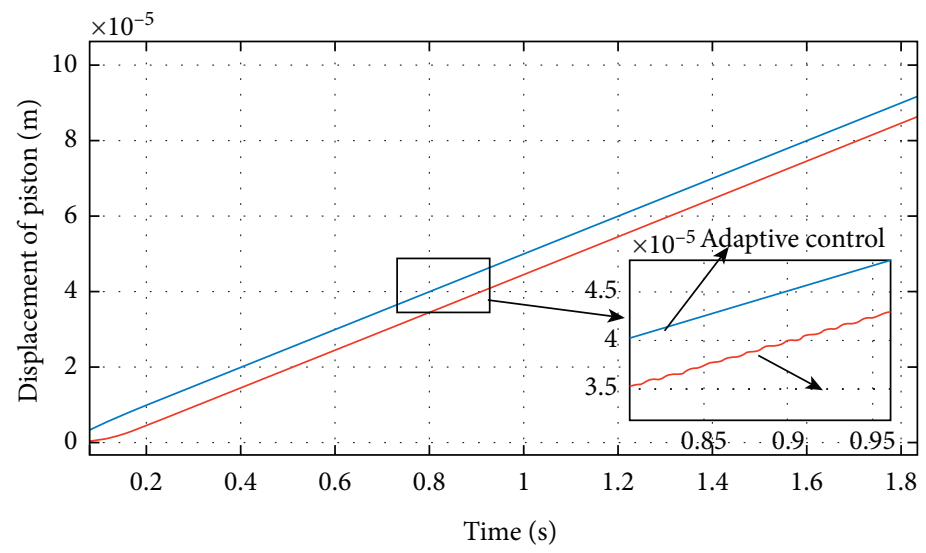

Figure 5: Piston displacement.

adaptive control are compared, as shown in Figure 5. The test results show that the system with PID control still crawls the phenomenon at low speed and has a large tracking error, which is due to the "stick-slip" phenomenon in nonlinear friction. The addition of adaptive friction compensation control can effectively eliminate the crawling phenomenon caused by nonlinear friction at the low speed of the digital hydraulic cylinder, which could improve the control accuracy of the system at low speed.

4.3. Speed Dead Zone and Position Truncation. Considering the triangle wave input, the triangle wave with a period of $0.8 \mathrm{~s}$ and a speed of $5 \times 10^{-5}(\mathrm{~m} / \mathrm{s})$ is used as the system input. The PID control and adaptive control position tracking errors are added, as shown in Figure 6.

Figures 6(a) and 6(b) show that the position curve with PID control added had obvious jitter and a large error, while the position curve with adaptive friction compensation control added has no obvious jitter, and the error is relatively small. Figure 6(c) shows that the position error with PID control is about $2 \times 10^{-6} \mathrm{~m}$, and the position error with adaptive friction compensation control is less than $6 \times 10^{-6} \mathrm{~m}$. Figure $6(\mathrm{a})$ shows that a dead zone could be observed in the position tracking system with PID control, and the rapid increase in position error leads to the phenomenon of "position truncation." The reason is that at the peak of the triangular waveform, the system needs to stop and reverse movement because of the effects of the maximum static friction; thus, when the system starts in the reverse direction, it needs to overcome the maximum static friction before starting, causing the actual position to lag behind the given signal resulting in a position error. Figure 6(d) further shows that under the PID control method when the position reaches the maximum value, the speed crosses zero, indicating a "velocity dead zone," and an irregular jitter in the crawling caused by the preslip and nonlinear friction force at the low-speed stage. The system with adaptive control can effectively suppress the "position truncation" and "speed dead zone" phenomena of the 


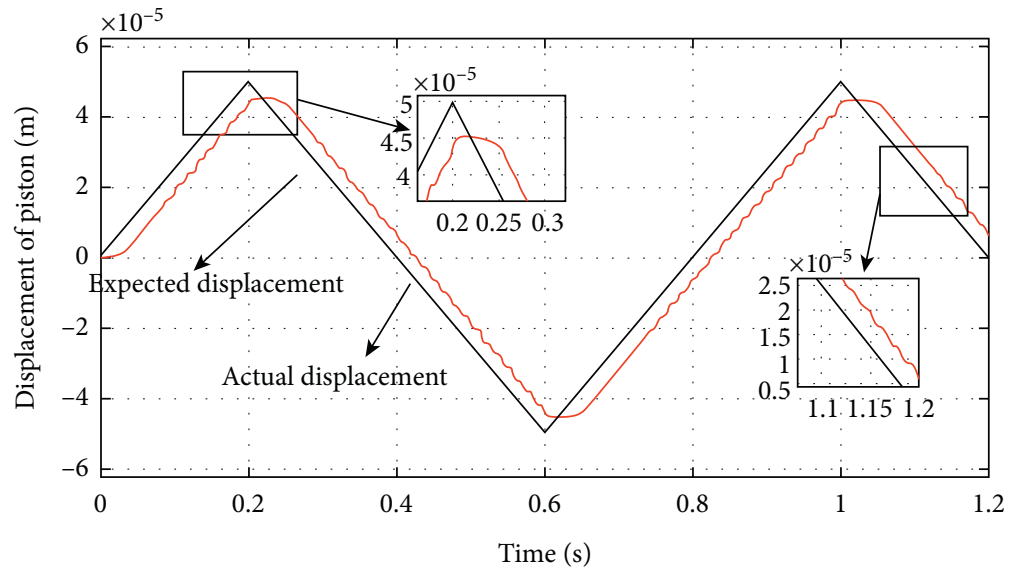

(a)

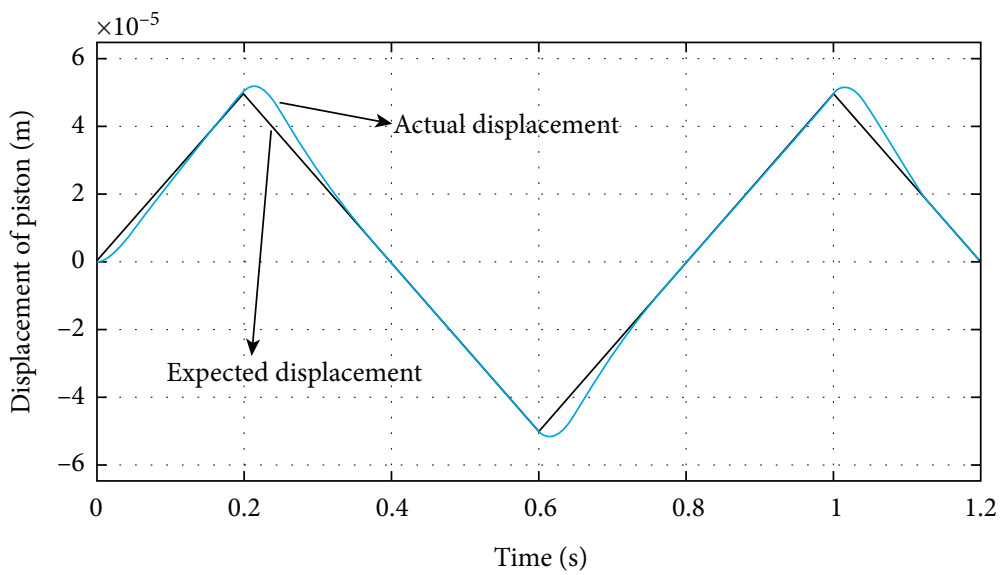

(b)

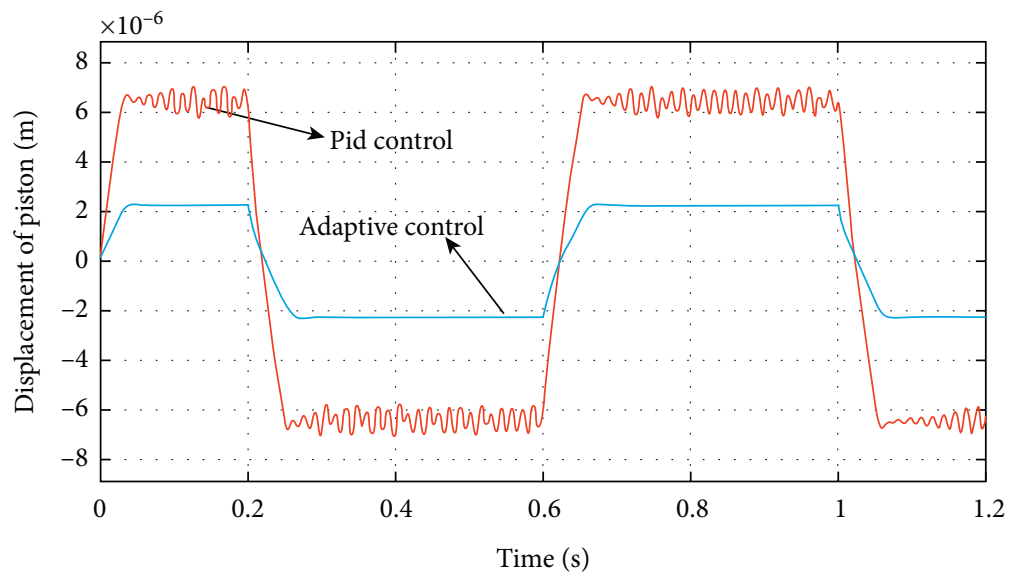

(c)

Figure 6: Continued. 


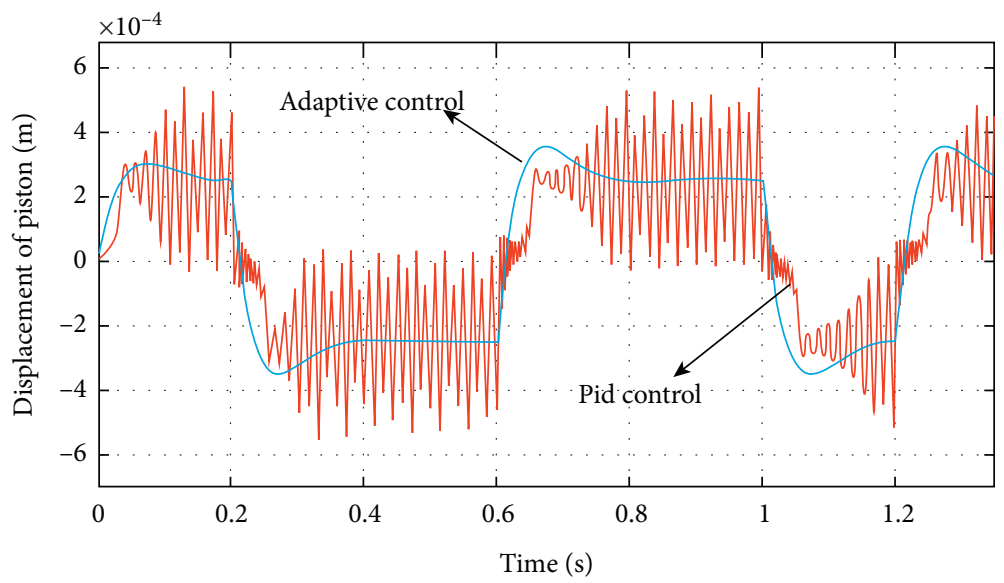

(d)

FIGURE 6: Comparison of results of different control methods: (a) no adaptive friction compensation system added; (b) add adaptive friction compensation system; (c) comparison of displacement errors between two control methods; (d) comparison of two control methods for piston speed.

system. Moreover, the position and speed of the system can track the command signal well while improving system stability.

\section{Conclusion}

This paper analyzes the effects of nonlinear friction on the performance of digital hydraulic cylinders, establishes a mathematical model of digital hydraulic cylinders with the LuGre friction model, and designs a dual-observer structure to estimate the unobservable state variables in the friction model. To address the parameters with inconsistent changes, an adaptive friction compensation control algorithm is designed, parameters in the friction model are estimated online, and a nonlinear friction is compensated online. The Lyapunov method is used to prove the stability of the closed-loop system using the adaptive friction compensation method. Finally, the MATLAB/Simulink is used to simulate the system performance. The test results show that because of the difference between the maximum static friction force and the Coulomb friction force in nonlinear friction, the system will have a large static difference or even a steady-state limit cycle oscillation phenomenon. The "stick-slip" phenomenon in the nonlinear friction will cause the lowspeed creeping phenomenon to occur in the system. The nonlinearity of the preslip and low-speed friction forces will cause the system's "position shaving" and "speed dead zone" phenomena. The abovementioned phenomenon can be improved effectively by adding an adaptive friction compensation control strategy. This control method has practical application value for improving the performance index of the digital hydraulic cylinder.

Future works should consider the unmodeled dynamics of the system, and the T-S fuzzy system should be used to approximate the unmodeled dynamics. At the same time, the adaptive adjustment fuzzy identification parameters should be considered and the Lyapunov function should be used to prove the stability of the control system and finally realize that the system output can track the expected output well.

\section{Conflicts of Interest}

The authors declare that they have no conflicts of interest.

\section{Acknowledgments}

This work was supported by the National Natural Science Foundation of China (51574140), China Postdoctoral Science Foundation (2020M682268 and 2020M672089), National Key Research and Development Plan of Ministry of Science and Technology of the People's Republic of China (2017YFC0804305), and Major Science and Technology Innovation Projects in Shandong Province (2019SDZY04).

\section{References}

[1] S. J. Xu, J. J. Lou, and L. K. Peng, "Nonlinear robust position tracking control of digital hydraulic cylinder with regard to uncertainties and disturbance," Journal of Naval University of Engineer, vol. 27, no. 2, pp. 74-80, 2015.

[2] J. Chen, J. F. Xing, and L. K. Peng, "Analysis and experiment on nonlinear dynamic characteristics of digital hydraulic cylinder," Mechanical Science and Technology for Aerospace Engineering, vol. 35, no. 7, pp. 1035-1042, 2016.

[3] B. Friedland and Y.-J. Park, "On adaptive friction compensation," IEEE Transactions on Automatic Control, vol. 37, no. 10, pp. 1609-1612, 1992.

[4] Y. P. Ren and S. Y. Li, "An approach for nonlinear PD controller design," Control Engineering of China, vol. 13, no. 3, pp. 224-229, 2005.

[5] Y. F. Wang and T. Y. Chai, "Adaptive fuzzy control method for dynamic friction compensation," Proceedings of the CSEE, vol. 25, no. 2, pp. 139-144, 2005.

[6] J. H. Hu, Y. L. Hou, Q. Gao, Y. Z. Chen, and S. P. Qu, "Slidingmode control for tank gun controlling system based on 
disturbance observer," Electronics Optics \&Control, vol. 25, no. 3, pp. 98-101, 2018.

[7] Y. Feng and X. Man, "Non-singular terminal sliding mode control of rigid manipulators," Automatica, vol. 38, no. 12, pp. 2159-2167, 2002.

[8] Y. Feng, S. Bao, and X. H. Yu, "Design method of non-singular terminal sliding mode control systems," Control and Decision, vol. 17, no. 2, pp. 194-198, 2002.

[9] S. B. Li, K. Q. Li, J. Q. Wang et al., "Nonsingular and fast terminal sliding mode control method," Information and Control, vol. 38, no. 1, pp. 1-8, 2009.

[10] S. C. Southward, C. J. Radcliffe, and C. R. MacCluer, "Robust nonlinear stick-slip friction compensation," Journal of Dynamic Systems, Measurement, and Control, vol. 113, no. 4, pp. 639-645, 1991.

[11] G. Liu, "Decomposition-based friction compensation using a parameter linearization approach," in Proceedings of the 2001 IEEE International Conference on Robotics \&Automation, Seoul, pp. 1155-1160, Seoul, Republic of Korea, May 2001.

[12] Q. W. Wang and Z. H. Liu, "Nonlinear friction decomposition control of flight motion simulator," Computer Simulation, vol. 23 , no. 7, pp. 275-279, 2006.

[13] Q. Liu, E. R. Lianjie, and J. K. Liu, "Overview of Charcteristics, Modeling and compensation of nonlinear friction in servo systems," Systems Engineering and Electrionics, vol. 24, no. 11 , pp. $45-52,2002$.

[14] A. Tesfaye, H. S. Ho Seong Lee, and M. Tomizuka, "A sensitivity optimization approach to design of a disturbance observer in digital motion control systems," IEEE/ASME Transactions on Mechatronics, vol. 5, no. 1, pp. 32-38, 2000.

[15] A. Yazdizadeh and K. Khorasani, "Adaptive friction compensation based on the Lyapunov scheme," in Proceedings of the 1996 IEEE International Conference on Control Applications, pp. 1060-1065, Dearborn, MI, USA, November 1996.

[16] H. Xiang, W. B. Tan, X. F. Li, and C. Y. Zhang, "Adaptive friction compensation based on LuGre model," Journal of Mechanical Engineering, vol. 48, no. 17, pp. 70-74, 2012.

[17] Z. S. Wang, Y. Wang, and B. K. Su, "An adaptive friction compensation method for high-precision turntable system," Journal of South China University of Technology, vol. 35, no. 9, pp. 55-59, 2007.

[18] S. I. Han, C. S. Jeong, and S. Y. Yang, "Robust sliding mode control for uncertain servo system using friction observer and recurrent fuzzy neural networks?" Journal of Mechanical Science and Technology, vol. 26, no. 4, pp. 1149-1159, 2012.

[19] S. Mobayen and F. Tchier, "Synchronization of A Class of uncertain chaotic systems with Lipschitz nonlinearities using state-feedback control design: a matrix inequality approach," Asian Journal of Control, vol. 20, no. 1, pp. 71-85, 2018.

[20] X. H. Chang, Y. Liu, and M. Q. Shen, "Resilient control design for lateral motion regulation of intelligent vehicle," IEEE/ ASMET Ransactions on Mechatronics, vol. 24, no. 6, pp. 2488-2497, 2019.

[21] Y. Wang, B. Niu, H. Wang, N. Alotaibi, and E. Abozinadah, "Neural network-based adaptive tracking control for switched nonlinear systems with prescribed performance: an average dwell time switching approach," Neurocomputing, vol. 435, no. 7, pp. 295-306, 2021.

[22] M. Li, H. Xin, X. D. Zhao, and D. Z. Guang, "Observer-based adaptive neural tracking control for output-constrained switched MIMO nonstrict-feedback nonlinear systems with unknown dead zone," Nonlinear Dynamics, vol. 99, no. 2, pp. 1019-1036, 2020.
[23] Q. Zhang, Z. G. Yuan, and D. Z. Xu, “An adaptivesecond order terminal sliding mode control for a class of uncertain nonlinear systems using disturbance observer," Control Theory \& Applications, vol. 34, no. 2, pp. 179-187, 2017.

[24] X. J. Wei and L. Y. Zhang, "Anti-disturbance control based on disturbance observer for a class of nonlinear strict-feedback systems," Control and Decision, vol. 31, no. 9, pp. 1697-1701, 2016.

[25] L. Xu and B. Yao, "Adaptive robust control of mechanical systems with non-linear dynamic friction compensation," International Journal of Control, vol. 81, no. 2, pp. 167-176, 2008.

[26] L. Liu, J. Chen, and J. F. Xing, "Research on transfer function of digital hydraulic fin stabilizer system," Hydraulic and Pneumatic, vol. 11, pp. 97-100, 2012. 